Original paper

\title{
Novel inverse planning optimization algorithm for robotic radiosurgery: First clinical implementation and dosimetric evaluation
}

\author{
Michele Zeverino $^{\mathrm{a}}$, Maud Marguet ${ }^{\mathrm{a}}$, Cedric Zulliger ${ }^{\mathrm{b}}$, André Durham ${ }^{\mathrm{b}}$, Raphael Jumeau ${ }^{\mathrm{b}}$, \\ Fernanda Herrera $^{\mathrm{b}}$, Luis Schiappacasse ${ }^{\mathrm{b}}$, Jean Bourhis ${ }^{\mathrm{b}}$, Francois O. Bochud ${ }^{\mathrm{a}}$, Raphael Moeckli ${ }^{\mathrm{a}, *}$ \\ ${ }^{a}$ Institute of Radiation Physics, Lausanne University Hospital and University of Lausanne, Lausanne, Switzerland \\ ${ }^{\mathrm{b}}$ Radiation-oncology Department, Lausanne University Hospital and University of Lausanne, Lausanne, Switzerland
}

A R T I C L E I N F O

\section{Keywords:}

Cyberknife

Inverse planning

SRS

SBRT

Plan comparison

\begin{abstract}
A B S T R A C T
Purpose: A novel optimization algorithm $\left(\mathrm{VOLO}^{\mathrm{TM}}\right)$ for robotic radiosurgery in the Precision ${ }^{\mathrm{TM}}$ treatment planning system was evaluated for different SRS/SBRT treatments and compared with the previous Sequential Optimization (SO) algorithm.

Materials and methods: Fifty cases of brain, spine, prostate and lung tumors previously optimized with SO, were re-planned with VOLO $^{\mathrm{rm}}$ algorithm keeping the same prescription, collimator type and size, optimization shells, and blocking structures. The dosimetric comparison involved target coverage, conformity (CI), gradient (GI) and homogeneity indexes, specific indicators of dose to OARs and number of nodes, beams, MU and delivery time. For brain only, plans were IRIS- and MLC-based (10 each). The remaining 30 plans were all IRIS-based.

Results: VOLO ${ }^{\mathrm{TM}}$ optimization was significantly superior for target coverage for prostate and spine, CI for brain, and for brain and urethra dose sparing. SO gave significantly better results for GI for prostate. VOLO ${ }^{\mathrm{TM}}$ showed a significantly steeper dose fall-off for brain MLC-based, while for prostate and spine SO was superior. For IRISbased plans, VOLO ${ }^{\mathrm{TM}}$ significantly reduced the nodes (36\%), beams (14\%), and MU (31\%). This led to an average reduction of delivery time of $20 \%$ (from $8 \%$ for brain to $30 \%$ for prostate). For MLC-based plans, VOLO ${ }^{\mathrm{TM}}$ significantly increased the nodes and beams (42\%) keeping the same number of MU. The averaged delivery time increased by $18 \%$.

Conclusions: With respect to SO, VOLO ${ }^{\mathrm{TM}}$ optimization algorithm provided better results in terms of delivery time for IRIS-based and of quality of dose distribution for MLC-based plans, respectively.
\end{abstract}

\section{Introduction}

Since its first clinical implementation in 1997 [1], the robotically steered radiosurgery machine Cyberknife ${ }^{\otimes}$ (Accuray Incorporated, USA) has been continuously upgraded to improve plan quality and delivery efficiency [2] through the introduction of technical features such as multi-purpose tracking methods, increased dose-rate, Monte-Carlo dose calculation algorithm [3], the variable collimator IRIS ${ }^{\mathrm{TM}}$ [4] and, most recently, the InCise ${ }^{\mathrm{TM}}$ multi-leaf collimator (MLC) [5]. The treatment planning system (TPS) underwent only one significant improvement when Precision ${ }^{\mathrm{TM}}$ replaced the existing version called MultiPlan ${ }^{\mathrm{TM}}$ in 2018. Although that upgrade improved the planning practice of the users, its inverse treatment planning algorithm, called Sequential Optimization (SO), remained the same.

The SO algorithm has been successfully used to design plans for a variety of SRS/SBRT treatments [6-10] although the result of the entire process can be significantly slow due to its intrinsic sequential nature. In fact, when large volumes are involved or complex dose distributions are required such as in simultaneous integrated boost (SIB) cases, each step requires a lengthy optimization, making the whole process slower. Typical optimization times for complex cases can easily take up to $4 \mathrm{~h}$.

Time reduction and beam reduction are two options available to reduce delivery time. Time reduction iteratively reduces the number of beams and nodes until the desired treatment time is achieved. Beam reduction re-optimizes the whole plan by minimizing the number of beams under a user-defined MU threshold. Both processes usually decrease the quality of the dose distribution.

The first optimization algorithm making use of graphical processing unit (GPU)-based dose calculation was introduced in 2012 for Tomotherapy (Accuray Incorporated, USA) treatment planning. It borrowed the same optimization algorithm already implemented in the Tomotherapy TPS, featuring a convolution/superposition dose

\footnotetext{
* Corresponding author at: Rue du Grand-Pré 1, 1007 Lausanne, Switzerland.

E-mail address: raphael.moeckli@chuv.ch (R. Moeckli).
} 
Table 1

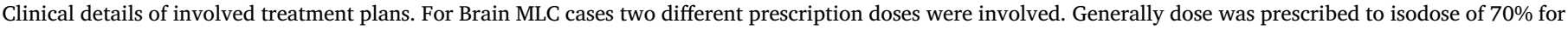
brain cases and $80 \%$ for all other cases.

\begin{tabular}{|c|c|c|c|c|c|c|}
\hline Disease & $\begin{array}{l}\text { PTV1 } \\
\text { cc }\end{array}$ & $\begin{array}{l}\text { PTV2 } \\
\text { cc }\end{array}$ & $\begin{array}{l}\text { Prescription } \\
\text { Dose } \\
\text { PTV1 } \\
\text { Gy/fractions }\end{array}$ & $\begin{array}{l}\text { Prescription } \\
\text { Dose } \\
\text { PTV2 } \\
\text { Gy/fractions }\end{array}$ & $\begin{array}{l}\text { Prescription } \\
\text { Isodose } \\
\%\end{array}$ & Tracking Method \\
\hline Brain IRIS & $\begin{array}{l}1.16 \\
(0.09-4.07)\end{array}$ & NA & $20 / 1$ & NA & 70 & 6D Skull \\
\hline Brain MLC & $\begin{array}{l}14.75 \\
(2.00-31.67)\end{array}$ & NA & $35 / 5$ and $33 / 3$ & NA & 80 & 6D Skull \\
\hline Lung & $\begin{array}{l}10.88 \\
(2.04-32.76)\end{array}$ & NA & $55 / 5$ & NA & 80 & Synchrony \\
\hline Spine & $\begin{array}{l}12.97 \\
(0.44-57.64)\end{array}$ & $\begin{array}{l}60.67 \\
(23.91-141.66)\end{array}$ & $35 / 5$ & $30 / 5$ & 80 & X-Sight Spine \\
\hline Prostate & $\begin{array}{l}2.99 \\
(0.68-6.16)\end{array}$ & $\begin{array}{l}84.13 \\
(44.32-145.96)\end{array}$ & $50 / 5$ & $36.25 / 5$ & 80 & Fiducials \\
\hline
\end{tabular}

calculation engine able to run on GPUs in order to decrease the computation time [11]. It relies on a single-cost function optimization and it uses an iterative least-squared minimization algorithm [12].

Recently, this GPU-based dose calculation engine was made available for Cyberknife ${ }^{\circledR}$ in the Precision $^{\mathrm{TM}}$ TPS with the name VOLO $^{\mathrm{TM}}$ as a way to reduce the computation time as well as the treatment time by using an improved sequencing algorithm. It can be used for all available collimators.

In this study, the VOLO ${ }^{\mathrm{TM}}$ planning performances were assessed with respect to the existing SO algorithm for four different SRS/SBRT typical clinical indications: brain metastasis, spine metastasis, lung and prostate cancer. Optimization algorithms were compared dosimetrically and in terms of delivery efficiency. Furthermore, a blinded clinical evaluation of rival plans was carried out to assess differences in quality of dose distributions.

\section{Materials and methods}

\subsection{Sequential Optimization (SO) and $V O L O^{\mathrm{TM}}$ algorithms employed}

SO was based on a multi-objective optimization that was executed sequentially as a series of individual optimization steps. Each step owned a single objective cost function that corresponded to a specific absolute constraint (that must not be violated) or soft constraint (i.e. a clinical goal with a relaxation value, the acceptable violation limit of the clinical goal). The user-defined sequence of steps replaced the definition of weighting factors used to prioritize objectives in conventional approaches. The optimization proceeded sequentially through the optimization steps, to build up the treatment plan one clinical objective at a time.

In this study, the maximum MU per beam or segment, node and plan, respectively, and maximum dose for each volume of interest (VOI) were used as absolute constraints. All other objectives controlling the DVH such as minimum dose for targets and maximum and mean dose for OARs were used as soft constraints. Our current practice for SObased plans involved the use of shell volumes of various diameter to improve dose conformality, and blocking structures such as eyes, metal dental artefacts, and arms to improve dose calculation and treatment delivery robustness. Dose conformality was controlled by assigning a soft constraint to the shell volumes with the exception of the most inner shell for for which an absolute constraint was set. A typical SO script is reported in Fig. 1 of Supplementary material. At the end of the SO process, both time and beam reduction were performed to achieve a treatment time ideally lower than $60 \mathrm{~min}$. MU threshold for beam reduction was set to six per fraction to neglect any possible small MU linearity issue. The last step involved a fine tuning of the dose distribution reducing $20 \%$ and $30 \%$ isodoses occurring in regions located apart from the target.
In $\mathrm{VOLO}^{\mathrm{TM}}$, objectives were optimized simultaneously and they were prioritized according to the relative penalties assigned to each objective. Therefore, a single multi-criteria cost function replaced the multiple single-criteria cost functions employed in SO. Available objectives were maximum dose, minimum dose and dose-volume objectives for both targets and OARs. Up to five objectives could be assigned to a single VOI with a specific penalty factor to indicate different priorities to the optimizer. No absolute constraints were available. Two specific parameters replaced both the time and beam reduction processes: total MU penalty and minimum MU per beam (or segment). The first one, ranging from 0 to 10 , meant to reduce the treatment time while the second one set the MU threshold for beams (or segments) per fraction.

In this study, maximum dose, minimum dose and multiple dosevolume objectives were used to optimize dose to both target and OAR according to the clinical goals. Relative penalties were initially set to 1 and then increased for a specific objective in order to achieve the desired goal.

In order to provide a consistent comparison, the VOLO ${ }^{\mathrm{TM}}$ optimization was performed by keeping the same arrangement as the SO plans, i.e. dose and isodose prescription, shell volumes, blocking structures, number and collimator sizes for IRIS-based plans, collimator type for MLC-based plans and minimum MU threshold per beam (6 per fraction). The total MU penalty was used instead of time and beam reduction processes to minimize the treatment time without compromising the dose distribution. Since no absolute constraint was available, maximum dose was used for all the shell volumes (see Fig. 2 of Supplementary material for details). As for SO plans, dose distribution was fine-tuned to reduce $20 \%$ and $30 \%$ isodoses out of the target volume.

For both algorithms, the whole process involved the beam optimization (node selection, beam size, beam weights) followed by the final dose calculation performed with either the Ray Tracing algorithm or Monte-Carlo independently from the collimator chosen.

Both algorithms considered delivery time as the summation of the patient setup time, beam-on-time (BOT), the time spent by the robotic arm to move between nodes, and the time required for imaging. That latter could be modified by the user according to the frequency of stereoscopic X-ray images chosen for the treatment. Since frameless stereotactic treatments were involved, the need for repeating images became essential and required up to $20 \%$ of the overall treatment time according to the tracking method involved. Treatment time depended upon the dose per fraction, the number and size of the targets, and the collimator type, size or aperture. For SO-based plans, typical values range from $20 \mathrm{~min}$ to more than $3 \mathrm{~h}[10,13,14]$.

\subsection{Patient selection and planning comparison}

A head-to-head quantitative comparison of dose distribution and 
Table 2

OARs clinical dose objectives for the different diseases and fractionations involved in the study. Spinal cord PRV $=$ Spinal cord $+5 \mathrm{~mm}$ isotropic margin

\begin{tabular}{|c|c|c|}
\hline OAR & Figure of merit & Clinical goal \\
\hline \multicolumn{3}{|l|}{ Brain (1 fraction) } \\
\hline Brain & V12Gy & $<10 \mathrm{cc}$ \\
\hline Brainstem & Dmax & $<15 \mathrm{~Gy}$ \\
\hline Chiasma & V8Gy & $<0.2 \mathrm{cc}$ \\
\hline \multirow[t]{2}{*}{ Optic Nerves } & V8Gy & $<0.2 \mathrm{cc}$ \\
\hline & Dmax & $<10 \mathrm{~Gy}$ \\
\hline Eyes & V8Gy & $<0.1 \mathrm{cc}$ \\
\hline Lenses & V1.5 Gy & $<0.1 \mathrm{cc}$ \\
\hline \multicolumn{3}{|l|}{ Brain (3 fractions) } \\
\hline Brain & V18Gy & $<1 \mathrm{cc}$ \\
\hline Brainstem & Dmax & $<23.1 \mathrm{~Gy}$ \\
\hline Chiasma & V15Gy & $<0.2 \mathrm{cc}$ \\
\hline Optic Nerves & V15Gy & $<0.2 \mathrm{cc}$ \\
\hline \multicolumn{3}{|l|}{ Brain (5 fractions) } \\
\hline Brainstem & Dmax & $<31 \mathrm{~Gy}$ \\
\hline Chiasma & Dmax & $<27 \mathrm{~Gy}$ \\
\hline Optic Nerves & Dmax & $<27 \mathrm{~Gy}$ \\
\hline \multicolumn{3}{|l|}{ Lung (5 fractions) } \\
\hline \multirow[t]{4}{*}{ Lungs-PTV } & V5Gy & $<50 \%$ \\
\hline & V12.5 Gy & $<1500 \mathrm{cc}$ \\
\hline & V13.5 Gy & $<1000 \mathrm{cc}$ \\
\hline & V20Gy & $<10 \%$ \\
\hline \multirow[t]{3}{*}{ Bronchus } & V18Gy & $<4 \mathrm{cc}$ \\
\hline & V35Gy & $<0.5 \mathrm{cc}$ \\
\hline & Dmax & $<38 \mathrm{~Gy}$ \\
\hline \multirow[t]{3}{*}{ Heart } & V32Gy & $<15 \mathrm{cc}$ \\
\hline & V40Gy & $<1 \mathrm{cc}$ \\
\hline & D0.5 cc & $<29 \mathrm{~Gy}$ \\
\hline \multirow[t]{2}{*}{ Big Vessels } & V47Gy & $<10 \mathrm{cc}$ \\
\hline & D0.5 cc & $<53 \mathrm{~Gy}$ \\
\hline \multirow[t]{3}{*}{ Esophagus } & V20Gy & $<10 \mathrm{cc}$ \\
\hline & V27.5 Gy & $<5 \mathrm{cc}$ \\
\hline & D0.5 cc & $<32 \mathrm{~Gy}$ \\
\hline \multirow[t]{3}{*}{ Ribs } & V30Gy & $<10 \mathrm{cc}$ \\
\hline & V35Gy & $<1 \mathrm{cc}$ \\
\hline & V43Gy & $<0.03 \mathrm{cc}$ \\
\hline \multicolumn{3}{|l|}{ Spine (5 fractions) } \\
\hline \multirow[t]{3}{*}{ Spinal Cord } & V20Gy & $<1.2 \mathrm{cc}$ \\
\hline & V22.5 Gy & $<0.25 \mathrm{cc}$ \\
\hline & $\mathrm{D} 0.03 \mathrm{cc}$ & $<25.3 \mathrm{~Gy}$ \\
\hline Spinal Cord PRV & V30Gy & $<0.1 \mathrm{cc}$ \\
\hline Roots & Dmax & $<33 \mathrm{~Gy}$ \\
\hline Esophagus & Dmax & $<35 \mathrm{~Gy}$ \\
\hline \multicolumn{3}{|c|}{ Prostate (5 fractions) } \\
\hline \multirow[t]{3}{*}{ Rectum } & D1cc & $<38 \mathrm{~Gy}$ \\
\hline & $\mathrm{D} 0.1 \mathrm{cc}$ & $<41 \mathrm{~Gy}$ \\
\hline & Dmax & $<44 \mathrm{~Gy}$ \\
\hline Sigmoid & V20Gy & $<10 \mathrm{cc}$ \\
\hline \multirow[t]{2}{*}{ Small Bowel } & V15Gy & $<10 \mathrm{cc}$ \\
\hline & Dmax & $<20 \mathrm{~Gy}$ \\
\hline \multirow[t]{3}{*}{ Urethra } & V39Gy & $<1 \mathrm{cc}$ \\
\hline & V41Gy & $<0.1 \mathrm{cc}$ \\
\hline & Dmax & $<43 \mathrm{~Gy}$ \\
\hline \multirow[t]{3}{*}{ Bladder } & V39Gy & $<3 \mathrm{cc}$ \\
\hline & V41Gy & $<1 \mathrm{cc}$ \\
\hline & V45Gy & $<0.1 \mathrm{cc}$ \\
\hline Penis Bulb & D3cc & $<35 \mathrm{~Gy}$ \\
\hline Anal Canal & D3cc & $<25 \mathrm{~Gy}$ \\
\hline Femoral Heads & V20Gy & $<10 \mathrm{cc}$ \\
\hline
\end{tabular}

delivery efficiency between SO and $\mathrm{VOLO}^{\mathrm{TM}}$ optimization algorithms was carried out for 50 patients encompassing the following diseases: brain, lung, spine and prostate. For brain, 20 patients were enrolled and equally split in two groups according to collimator employed: Brain IRIS and Brain MLC. Lung, spine and prostate cases $(n=10$ per each group) were all IRIS-based plans. Spine and prostate cases were SIB cases. The distributions of the target size as well as different prescription doses are given in Table 1.
Table 3

Dosimetric comparison for target volumes listed per different disease. Statistically significant differences are reported in bold. * ns: not significant.

\begin{tabular}{|c|c|c|c|}
\hline Parameter & Sequential Optimization & VOLO $^{\mathrm{TM}}$ & p-value \\
\hline \multicolumn{4}{|l|}{ Brain IRIS } \\
\hline TVPIV (\%) & $0.98 \pm 0.01$ & $0.99 \pm 0.01$ & $\mathrm{~ns}^{*}$ \\
\hline GI & $4.76 \pm 0.99$ & $4.41 \pm 0.84$ & $<0.05$ \\
\hline CI & $0.72 \pm 0.11$ & $0.77 \pm 0.11$ & $<0.01$ \\
\hline $\mathrm{nHI}$ & $1.07 \pm 0.07$ & $1.03 \pm 0.04$ & ns \\
\hline \multicolumn{4}{|l|}{ Brain MLC } \\
\hline TVPIV (\%) & $0.98 \pm 0.02$ & $0.99 \pm 0.01$ & ns \\
\hline GI & $3.00 \pm 0.34$ & $3.03 \pm 0.53$ & ns \\
\hline CI & $0.83 \pm 0.09$ & $0.87 \pm 0.07$ & $<0.005$ \\
\hline $\mathrm{nHI}$ & $1.08 \pm 0.09$ & $1.09 \pm 0.05$ & ns \\
\hline \multicolumn{4}{|l|}{ Lung } \\
\hline TVPIV (\%) & $0.92 \pm 0.11$ & $0.92 \pm 0.11$ & $\mathrm{~ns}$ \\
\hline GI & $4.54 \pm 0.92$ & $4.31 \pm 0.67$ & ns \\
\hline CI & $0.83 \pm 0.09$ & $0.83 \pm 0.09$ & ns \\
\hline $\mathrm{nHI}$ & $1.16 \pm 0.22$ & $1.04 \pm 0.27$ & ns \\
\hline \multicolumn{4}{|l|}{ Prostate } \\
\hline TVPIV1 (\%) & $0.96 \pm 0.04$ & $0.99 \pm 0.01$ & $<0.02$ \\
\hline TVPIV2 (\%) & $0.96 \pm 0.05$ & $0.98 \pm 0.03$ & ns \\
\hline GI & $3.67 \pm 0.24$ & $4.05 \pm 0.32$ & $<0.02$ \\
\hline CI1 & $0.72 \pm 0.21$ & $0.68 \pm 0.07$ & ns \\
\hline $\mathrm{CI} 2$ & $0.84 \pm 0.05$ & $0.85 \pm 0.03$ & ns \\
\hline nHI1 & $1.09 \pm 0.11$ & $1.07 \pm 0.07$ & $\mathrm{~ns}$ \\
\hline nHI2 & $1.59 \pm 0.33$ & $1.56 \pm 0.11$ & ns \\
\hline \multicolumn{4}{|l|}{ Spine } \\
\hline TVPIV1 (\%) & $0.92 \pm 0.07$ & $0.92 \pm 0.07$ & ns \\
\hline TVPIV2 (\%) & $0.81 \pm 0.17$ & $0.92 \pm 0.08$ & $<0.05$ \\
\hline GI & $4.55 \pm 0.87$ & $4.26 \pm 0.74$ & ns \\
\hline CI1 & $0.53 \pm 0.21$ & $0.52 \pm 0.24$ & $\mathrm{~ns}$ \\
\hline $\mathrm{CI} 2$ & $0.65 \pm 0.22$ & $0.74 \pm 0.05$ & $\mathrm{~ns}$ \\
\hline nHI1 & $1.21 \pm 0.14$ & $1.15 \pm 0.08$ & ns \\
\hline nHI2 & $1.59 \pm 0.33$ & $1.48 \pm 0.20$ & ns \\
\hline
\end{tabular}

Table 4

Dosimetric comparison for OARs. Only statistically significant results are reported.

\begin{tabular}{llllll}
\hline OAR & $\begin{array}{l}\text { Figure } \\
\text { of } \\
\text { merit }\end{array}$ & Clinical goal & $\begin{array}{l}\text { Sequential } \\
\text { Optimization }\end{array}$ & VOLO $^{\mathrm{TM}}$ & p value \\
& & & & & \\
Brain IRIS & & & & & \\
Brain & V12Gy & $<10 \mathrm{cc}$ & $4.8 \pm 4.6 \mathrm{cc}$ & $4.1 \pm 3.9 \mathrm{cc}$ & $<0.001$ \\
& Dmean & & $0.6 \pm 0.4 \mathrm{~Gy}$ & $0.5 \pm 0.4 \mathrm{~Gy}$ & $<0.001$ \\
Brainstem & Dmax & $<15 \mathrm{~Gy}$ & $2.4 \pm 3.0 \mathrm{~Gy}$ & $2.9 \pm 3.2 \mathrm{~Gy}$ & $<0.01$ \\
Brain $M L C$ & & & & & \\
Brainstem & Dmax & $<23.1 \mathrm{~Gy}$ & $6.9 \pm 7.0 \mathrm{~Gy}$ & $6.0 \pm 6.9 \mathrm{~Gy}$ & $<0.02$ \\
o & & & & & \\
Urethra & & & & & \\
& V39Gy & $<1 \mathrm{cc}$ & $0.3 \pm 0.3 \mathrm{cc}$ & $0.1 \pm 0.2 \mathrm{cc}$ & $<0.01$ \\
& V41Gy & $<0.1 \mathrm{cc}$ & $0.04 \pm 0.05 \mathrm{cc}$ & $0.01 \pm 0.03 \mathrm{cc}$ & $<0.001$ \\
& Dmax & $<43 \mathrm{~Gy}$ & $40.2 \pm 1.1 \mathrm{~Gy}$ & $38.1 \pm 1.2 \mathrm{~Gy}$ & $<0.005$
\end{tabular}

All patients were previously treated in our department with Cyberknife and were planned with SO algorithm using the IRIS or MLC collimator. For IRIS-based plans, up to five collimator sizes per target were employed ranging between $7.5 \mathrm{~mm}$ and $60 \mathrm{~mm}$ according to the target size. No aperture restrictions were adopted for MLC-based plans.

Generally, the $98 \%$ of the planning target volume (PTV) did receive the prescription dose unless its coverage compromised OAR dose limits. Clinical dose goals for the OARs involved in the comparison are reported in Table 2.

Raytracing and Monte-Carlo dose calculation algorithms were equally available for both optimizations algorithms. Raytracing was used for brain and prostate, while for spine the choice of the calculation algorithm depended upon the target localization with respect to low density VOI. For lung cases, the Monte-Carlo was employed for final dose calculation. 


\section{Delivery parameters}

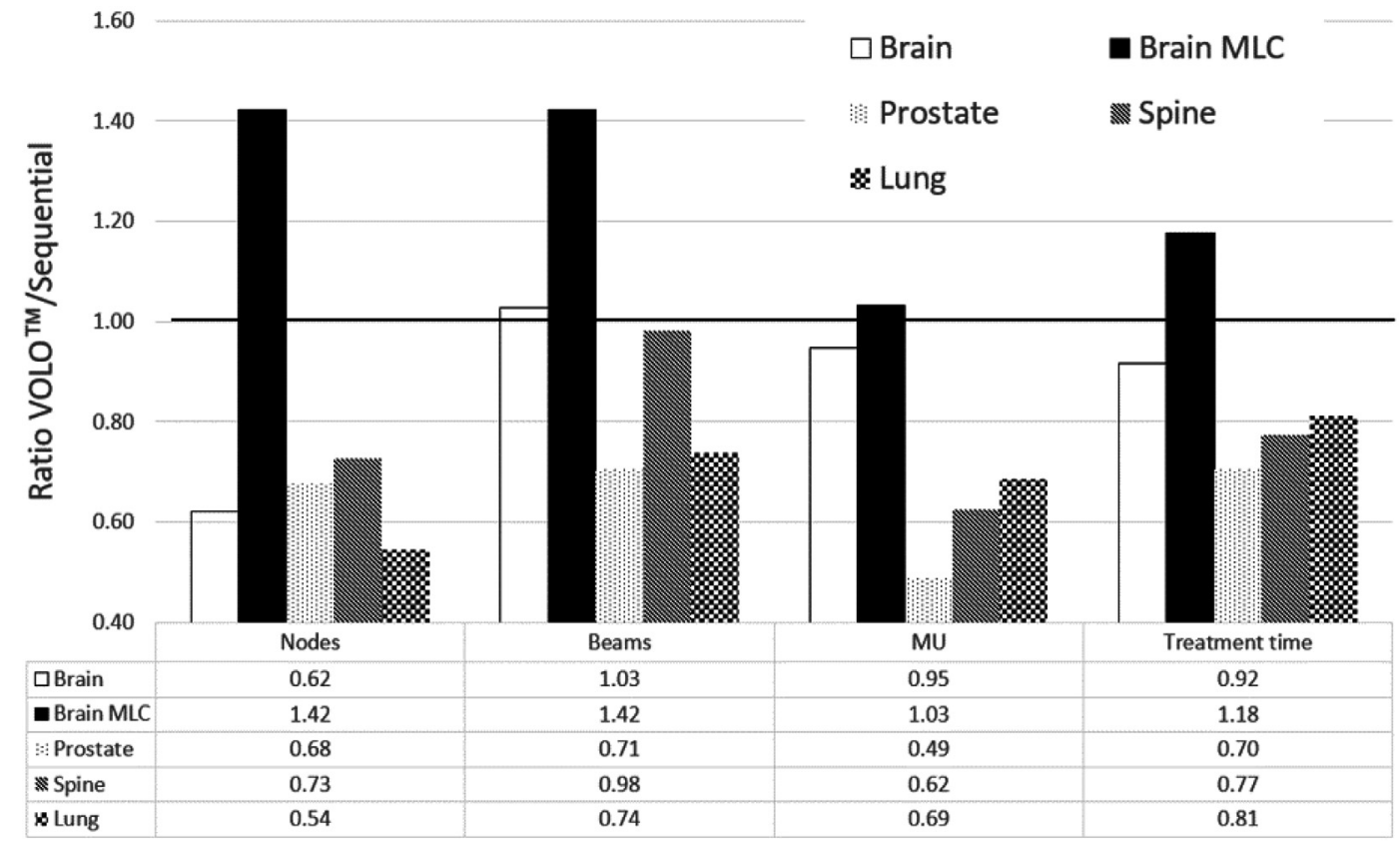

Fig. 1. Summary of delivery parameters listed per different disease. Values are reported as the ratio between VOLO ${ }^{\mathrm{TM}}$ and SO optimization algorithms.

Plans were dosimetrically compared in terms of PTV receiving the prescription isodose volume ( $\mathrm{PTV}_{\mathrm{PIV}}$ ), gradient index (GI), conformity index (CI), normalized homogeneity index (nHI), peripheral dose falloff and dose to OARs. CI and GI were calculated according to their original formulations $[15,16]$. Concerning the target homogeneity, a new formulation was introduced in order to provide an index independent from the different prescription isodoses of plans involved in the study: nHI was computed as (D1\%/D99\%)/PI, where PI is the prescription percentage isodose (70\% or $80 \%$ according to the disease). Peripheral dose fall-off was assessed to evaluate the volume of the $90 \%$, $80 \%, 20 \%$ and $10 \%$ isodoses.

The delivery efficiency of plans was evaluated by comparing the number of nodes, beams, MU and treatment time. For IRIS-based plans, the average collimator size $(\bar{C})$ and the MU-weighted average collimator size $\left(\bar{C}_{M U}\right)$ were also computed to investigate the differences in sequencing between algorithms according to:

$\bar{C}=\frac{\sum_{i} C_{i}}{N}$

and

$\bar{C}_{M U}=\frac{\sum_{i} M U_{i} C_{i}}{\sum_{i} M U_{i}}$

where $\mathrm{i}$ runs over the number of beams and $\mathrm{N}$ is the total number of beams.

For the same purpose, the MLC-based plans were investigated assessing the number of MU per beam as the MLC aperture was not directly controlled by the user.

\subsection{Plan evaluation and scoring}

For each investigated parameter, the Wilcoxon matched-paired signed-rank test with a significance level of $p=0.05$ was used to assess significant differences for both optimization algorithms.

The plan quality was also assessed by experienced radio-oncologists each specialized in SRS/SBRT specific pathology in a blinded clinical evaluation of plans. The radio-oncologists were asked to score the difference between $\mathrm{SO}$ and $\mathrm{VOLO}^{\mathrm{TM}}$ plans of a given case on a scale between 1 and 5, where 1 was strongly lower, 3 equal and 5 strongly better. The scoring was asked to be based on target coverage and conformity, OAR sparing, and peripheral dose fall-off. Initially, no information was given about the treatment time. At the end of the scoring process, physicians were asked to reconsider their choice while taking treatment time into account.

\section{Results}

Plans optimized with VOLO $^{\mathrm{TM}}$ were dosimetrically comparable to plans optimized with SO except for some statistically significant differences. Concerning target volumes, for brain metastasis plans, VOLO ${ }^{\mathrm{TM}}$ returned a better $\mathrm{GI}$ and $\mathrm{CI}$ with respect to SO. VOLO ${ }^{\mathrm{TM}}$ also improved target coverage of SIB large PTV for prostate and SIB boost PTV for spine metastasis plans. On the other hand, SO returned a better GI for prostate cases. Dose differences are reported in detail in Table 3.

For OARs, VOLO ${ }^{\mathrm{TM}}$ was better in terms of organ sparing of the brain and urethra while maximum dose to brain stem was reduced with SO for IRIS-based plans and with VOLO ${ }^{\mathrm{TM}}$ for MLC-based plans. Statistically significant results are reported in Table 4 while all other results are reported in Table 1 of Supplementary material.

For IRIS-based plans, VOLO ${ }^{\mathrm{TM}}$ optimization significantly reduced the number of nodes, beams, and MU. Overall, the $\mathrm{VOLO}^{\mathrm{TM}} / \mathrm{SO}$ ratio was $0.64,0.86$. and 0.69 , respectively. This ratio varied between classes of plans and results are summarized in Fig. 1 and in more details in Table 2 of Supplementary material. This improvement in delivery efficiency led to an overall reduction of treatment time of $20 \%$ with respect to SO-optimized plans. The maximum reduction of average treatment time was observed for prostate plans (30\%) while for brain metastasis plans the average reduction was $8 \%$ only.

For MLC-based plans, VOLO ${ }^{\mathrm{m}}$ optimization significantly increased the number of nodes and beams, keeping the same ratio 1:1 as observed for SO plans. The number of MU slightly increased with $\mathrm{VOLO}^{\mathrm{TM}}(3 \%)$ therefore the number of $\mathrm{MU}$ per beam resulted significantly lower than SO. As a result, $\mathrm{VOLO}^{\mathrm{TM}}$ plans resulted in average longer than SO plans in terms of delivery time (18\%). Nonetheless, due to the low extent of delivery time for such plans the absolute difference in terms of delivery time was 4 minutes only. 

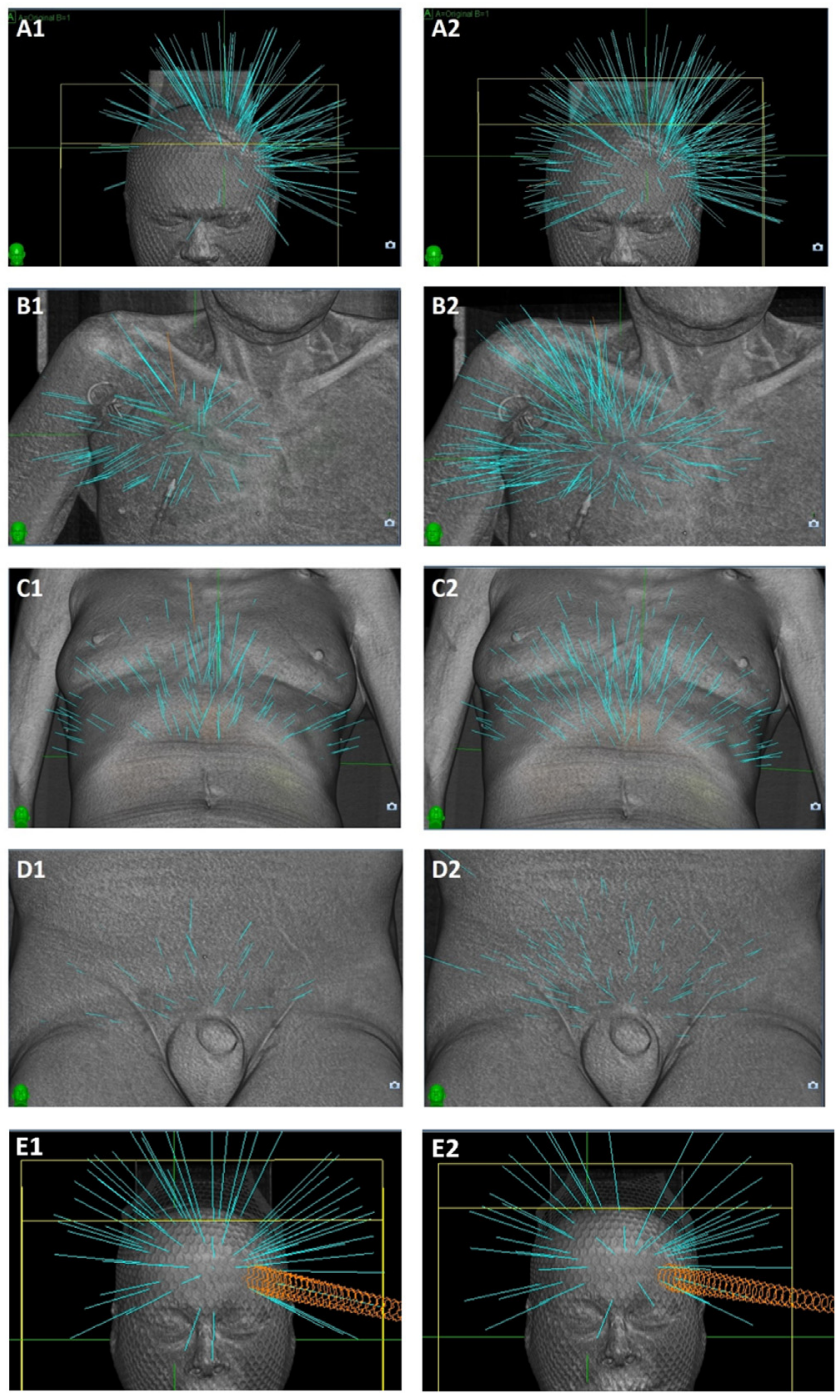

Fig. 2. 3D reconstructions of treatment delivery for A. Brain IRIS, B. Lung, C. Spine, D. Prostate and E. Brain MLC. Beams are depicted with blue lines. Left side $\left(\right.$ VOLO $^{\mathrm{Tm}}$ algorithm), right side (SO algorithm).

Three-dimensional reconstructions of delivery geometries for both $\mathrm{SO}$ and $\mathrm{VOLO}^{\mathrm{TM}}$ optimization are reported in Fig. 2. It shows the reduction of treatment beams for IRIS-based $\mathrm{VOLO}^{\mathrm{TM}}$ plans and the increase of treatment beams for MLC-based VOLO $^{\mathrm{TM}}$ plans.

Fig. 3 plots the peripheral dose fall-off for each class of plan. The $\mathrm{VOLO}^{\mathrm{TM}} / \mathrm{SO}$ ratio was observed to vary significantly between groups of plans. For both prostate and spine metastasis plans the ratio was above the unity, for brain IRIS metastasis it was below the unity reaching the lowest value for low doses for brain MLC metastasis while for lung it was below unity for high and medium doses and above unity for low doses.

Mean $\bar{C}$ and $\bar{C}_{M U}$ values are reported in Table 5 . VOLO ${ }^{\mathrm{TM}}$ plans made use of larger collimator size for all plan classes except brain metastasis. The VOLO ${ }^{\mathrm{TM}} / \mathrm{SO}$ ratio for $\bar{C}$ ranged between 1 and 1.41 for brain metastasis and prostate, respectively. The $\bar{C} / \bar{C}_{M U}$ ratio was larger for $\mathrm{VOLO}^{\mathrm{TM}}$ plans with respect to SO plans suggesting the use of an increased MU fraction for smaller collimator by the $\mathrm{VOLO}^{\mathrm{TM}}$ optimization algorithm.

For brain MLC, the VOLO ${ }^{\mathrm{TM}} / \mathrm{SO}$ ratio of the MU/beam was 0.73 (115 $\mathrm{MU} /$ beam and $158 \mathrm{MU} /$ beam for VOLO ${ }^{\mathrm{TM}}$ and SO, respectively).

All VOLO $^{\mathrm{TM}}$ plans were considered clinically acceptable by radiooncologists. Overall, plans were judged to be clinically equivalent in
$22 \%$ of cases, VOLO ${ }^{\mathrm{TM}}$ plans were preferred in $60 \%$ of cases, and SO plans in the remaining $18 \%$. Clinical scores are summarized for each group of plans in Fig. 4. VOLO ${ }^{\mathrm{TM}}$ plans were generally preferred for all groups of plans except for lung where SO plans were considered clinically superior in $60 \%$ of cases.

Once the treatment time was taken into account, all plans formerly considered equivalent were re-scored as $\mathrm{VOLO}^{\mathrm{TM}}$ preferred for IRISbased plans and as SO for MLC-based plans. No changes were observed among formerly SO preferred plans. Overall, once the delivery time was taken into account VOLO $^{\mathrm{TM}}$ plans were preferred in $78 \%$ of cases $(77.5 \%$ and $80 \%$ for IRIS- and MLC-based plans, respectively.

\section{Discussion}

The VOLO ${ }^{\mathrm{TM}}$ optimization algorithm performances were tested for a sample of 50 SRS/SBRT plans with respect to the Sequential Optimization algorithm for two different collimator types: IRIS and MLC. Plan comparison involved the head-to-head evaluation of both dosimetric and delivery parameters for 4 typical clinical indications: brain, lung, prostate and spine.

VOLO $^{\mathrm{TM}}$ produced clinically acceptable plans well within the dosevolume clinical goals. A few statistically significant dosimetric differences were observed between algorithms. Instead, a major statistically significant difference was observed in terms of delivery parameters that was also strongly dependent on to the choice of the collimator type (IRIS or MLC).

Due to the retrospective nature of the study, it was difficult to assess differences in optimization time. However, the optimization time was sensibly reduced for $\mathrm{VOLO}^{\mathrm{Tm}}$ and it could be estimated up to ten times lower especially for SIB cases such as spine and prostate.

For IRIS-based plans, this study demonstrated the capability of the $\mathrm{VOLO}^{\mathrm{TM}}$ algorithm to significantly reduce the number of beams involved in the optimization process together with the number of total MU and, as a consequence, the treatment time. If, on the one hand, this may mitigate difficulties associated with stringent immobilization devices and long delivery times [17], on the other hand it may deteriorate the resulting dose distribution in terms of target coverage and peripheral dose fall-off.

In particular, for SRS/SBRT in which high fractional doses are applied, the biological effects over normal tissue complications and tumor control are more critical than those studied for standard fractionate radiotherapy [18]. It was therefore important to carefully evaluate the dosimetric impact of this newly introduced optimization algorithm for robotic stereotactic treatments.

According to our results, the reduced number of beams did not deteriorate the target conformality. On the contrary, VOLO ${ }^{\mathrm{TM}}$ demonstrated better target conformality than SO for brain metastasis and integrated boosts for prostate and spine metastasis plans and comparable results for lung cases. This demonstrated how the reduced number of beams was still enough to provide an optimal target conformality. Furthermore, there was no impact on the coverage or dose homogeneity of the target volume, independently from the class of the plan.

Reducing the beam number also did not influence the dose to OARs; it was comparable to SO optimization and, in some cases, even better. It is worth noting how $\mathrm{VOLO}^{\mathrm{TM}}$ performed better than SO when high steep dose gradients were involved. This was particularly observed in prostate plans where the urethra received significantly less dose than SO plans. In these plans, the urethra was often placed in proximity of the SIB target volume whose dose prescription was $50 \mathrm{~Gy}$. VOLO ${ }^{\mathrm{TM}}$ plans were able to increase urethra sparing without compromising the adjacent target volume coverage. This may reduce RT-associated late urinary toxicity [19].

Although $\mathrm{VOLO}^{\mathrm{rm}}$ plans were designed to keep the same configuration of collimator size used in the SO plans, the analysis of the distribution of the collimator size $(\bar{C})$ showed how in $\mathrm{VOLO}^{\mathrm{TM}}$ a larger 


\section{Peripheral Dose Falloff}

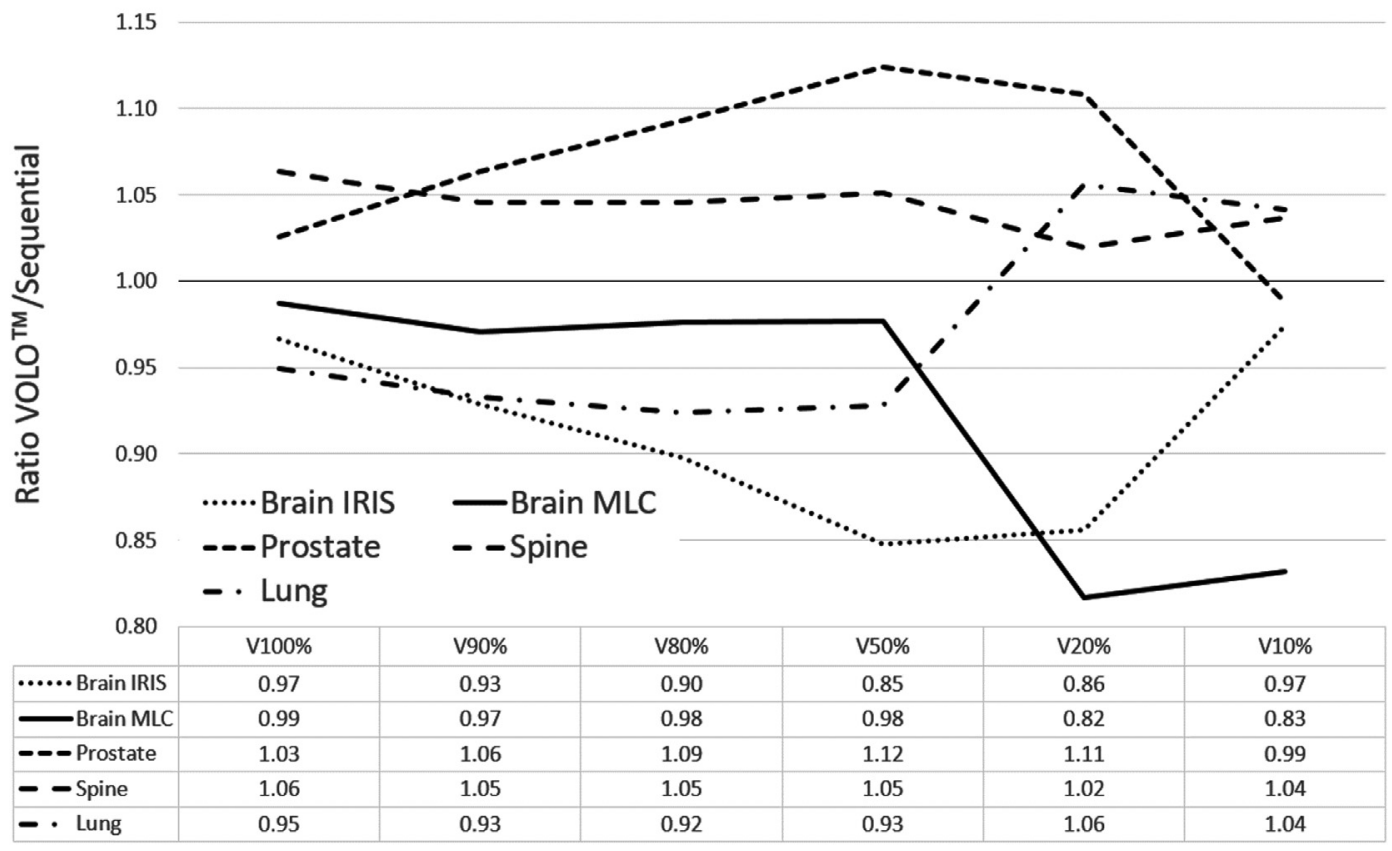

Fig. 3. Peripheral dose fall-off for different disease and overall averaged. Results are plotted as the ratio between VOLO and SO outcomes.

Table 5

Comparison of mean collimator size $(\bar{C})$ and MU-weighted mean collimator size $\left(\bar{C}_{M U}\right)$. Range is reported in brackets.

\begin{tabular}{|c|c|c|c|c|c|c|c|}
\hline & \multicolumn{2}{|l|}{$\bar{C}$} & \multicolumn{2}{|l|}{$\bar{C}_{M U}$} & \multirow{2}{*}{$\frac{\bar{C}}{\mathrm{VOLO}^{\mathrm{TM}} / \mathrm{SO}}$} & \multirow{2}{*}{$\begin{array}{l}\bar{C} / \bar{C}_{M U} \\
\text { SO }\end{array}$} & \multirow{2}{*}{$\frac{\bar{C} / \bar{C}_{M U}}{\mathrm{VOLO}^{\mathrm{TM}}}$} \\
\hline & SO & VOLO $^{\mathrm{TM}}$ & SO & VOLO $^{\mathrm{TM}}$ & & & \\
\hline Brain MTS & $9.4(7.5-12.5)$ & $9.4(7.5-12.5)$ & $9.4(7.5-12.5)$ & $9.3(7.5-12.5)$ & 1 & 1.000 & 1.011 \\
\hline Lung & $17.8(13.3-22.1)$ & $20.6(14.4-26.9)$ & $17.7(13.5-22.1)$ & $20.5(14.5-26.6)$ & 1.16 & 1.006 & 1.005 \\
\hline Spine MTS & 14.7 (8.0-19.7) & $19.8(10.0-28.8)$ & $14.4(7.9-19.4)$ & $19.0(10.0-27.6)$ & 1.35 & 1.021 & 1.042 \\
\hline Prostate & $17.6(12.5-21.4)$ & $24.8(19.7-28.7)$ & $17.5(13.5-20.9)$ & $24.2(17.8-28.4)$ & 1.41 & 1.005 & 1.024 \\
\hline
\end{tabular}

number of beams used a larger collimator size than in SO plans. The output factor of collimator sizes used in this study (range $7.5 \mathrm{~mm}-30 \mathrm{~mm}$ ) varies sensibly due to the small field size [20]. The use of a larger collimator size was the main factor enabling treatment time reduction due to the combination of both increased output factor and increased volume of target irradiated. The $\bar{C}$ ratio was higher for prostate and spine metastasis plans where larger collimator sizes were available in the optimization due to larger target volumes involved. This explains the deterioration of the quality of the dose distributions in terms of peripheral dose fall-off. Prostate plans that involved the use of the largest collimator sizes returned the worst results of dose fall-off when compared to SO plans. This phenomenon was not observed for brain cases since the target volumes were relatively small and so the collimator sizes involved in the optimization ranged only from $7.5 \mathrm{~mm}$ to $12.5 \mathrm{~mm}$. In case of large brain lesions where large collimator sizes are used, this may led to an increased risk of developing brain radionecrosis [21].

When large collimator sizes are involved, a possible option could be to decrease the collimator sizes for VOLO ${ }^{\mathrm{TM}}$ to improve peripheral dose fall-off.

The $\bar{C} / \bar{C}_{M U}$ ratio was found to be higher for $\mathrm{VOLO}^{\mathrm{TM}}$ than for SO for all plan classes. In SO plans, this ratio was close to unity, hence the MUs were equally distributed over the different collimator sizes available for plan optimization. Instead, for VOLO ${ }^{\mathrm{TM}}$ plans this ratio was superior to one, suggesting the increase of beam weighting for small collimator sizes in order to compensate the increased use of larger collimator sizes.

For lung VOLO ${ }^{\mathrm{TM}}$ plans, the peripheral dose fall-off was higher than
SO plans only for low doses (i.e. $10 \%$ and $20 \%$ of the prescription dose). Lung patients were treated while supine with arms placed along the body in order to provide the patient with the most comfortable position to be kept for the entire duration of the treatment. No fixed immobilization devices were used and so the arms may have moved with respect to the planned position. This meant that entering through the arms was avoided in the planning process to minimize the effect of arm positioning. That reduced the solid angle available for beam selection and the optimizer was forced to find alternative and sub-optimal paths often entering far from the target volume. Generally, such sub-optimal beams have a large number of MU because of their distance from PTV increasing the entrance dose. Due to the limited number of beams available, this effect was magnified for VOLO ${ }^{\mathrm{TM}}$ plans leading to an increase of low dose bath. Indeed, $\mathrm{VOLO}^{\mathrm{TM}}$ lung plans were only judged superior or equivalent to SO plans in $10 \%$ and $30 \%$ of the cases, respectively. In the remaining $60 \%$ of the cases, SO was judged superior because of the aforementioned effect.

MLC-based plan comparison was limited to brain metastasis as this is the main indication where this collimator is used in our institute. Although this can represent a limitation, it should be highlighted that $\mathrm{VOLO}^{\mathrm{TM}}$ plans were found dosimetrically superior to SO plans. The new optimization algorithm made use of an increased number of beams in order to achieve better dose distributions overall. In particular, $\mathrm{VOLO}^{\mathrm{TM}}$ plans solved the issue related to the large volumes of low doses that typically affected MLC-based SO plans. In principle, MLC is used when large tumor sizes are involved according to a SBRT approach. In this case, delivery time is usually below $30 \mathrm{~min}$. Therefore, a small 


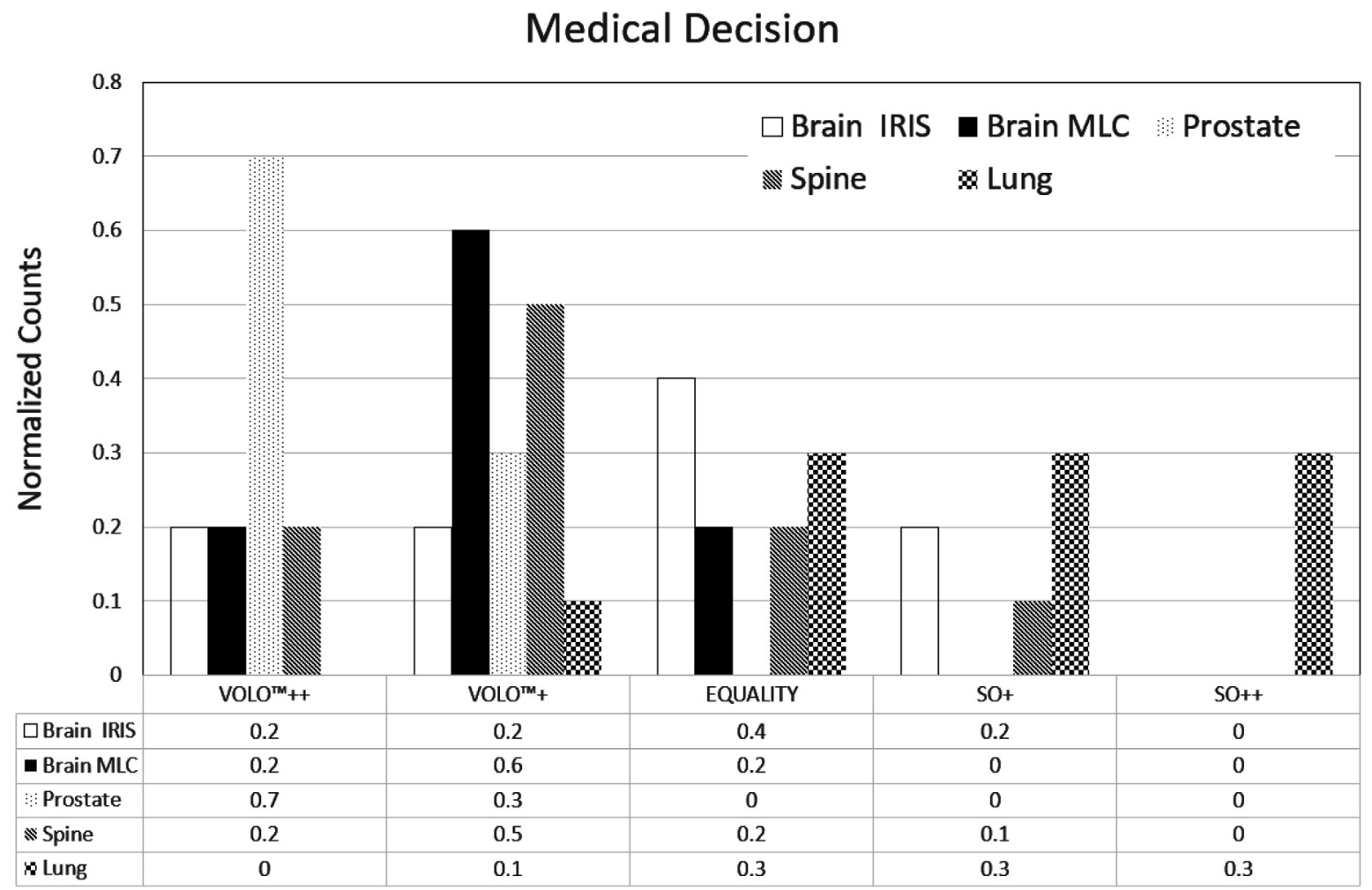

Fig. 4. Clinical evaluation of rival plans. $(++)$ means a strong preference and $(+)$ a fair preference. Delivery time was not a parameter taken into account at this stage.

increment of delivery time of a few minutes can be considered acceptable if a consistent improvement of the dose distribution can be achieved. However, further studies are needed to provide consistent comparisons for clinical indications other than brain metastasis.

Overall, the clinical evaluation only slightly preferred VOLO ${ }^{\mathrm{TM}}$ plans as long as treatment time was not taken into account in the scoring process. Once this parameter was taken into account, VOLO ${ }^{\mathrm{TM}}$ plans were chosen in almost $80 \%$ of the 50 plans, thus demonstrating how this new optimization algorithm provided an improvement in robotic radiosurgery treatment from a clinical perspective.

\section{Conclusion}

This study evaluated the performances of the $\mathrm{VOLO}^{\mathrm{TM}}$ optimization algorithm for robotic radiosurgery in comparison with the previously existing Sequential Optimization algorithm. Treatment time reduction was significant with VOLO $^{\mathrm{TM}}$ optimization for IRIS-based plans. The reduced number of beams involved in the delivery did not affect target coverage and dose conformity. However, peripheral dose fall-off increased in particular when large collimator sizes were involved. For MLC-based plans, VOLO ${ }^{\mathrm{TM}}$ optimization algorithm produced significantly better dose distributions for brain metastasis at the cost of a small increase in delivery time due to an increased number of beams involved.

\section{Appendix A. Supplementary data}

Supplementary data to this article can be found online at https:// doi.org/10.1016/j.ejmp.2019.07.020.

\section{References}

[1] Adler Jr JR, Chang SD, Murphy MJ, Doty J, Geis P, et al. The Cyberknife: A frameless robotic system for radiosurgery. Stereotact Funct Neurosurg 1997;69:124-8. [2] Kilby W, Dooley JR, Kuduvalli G, Sayeh S, Maurer Jr CR. The Cyberknife ${ }^{\circledR}$ robotic radiosurgery system in 2010. Technol Cancer Res Treat 2010;9(5):433-52.

[3] Ma C-M, Li JS, Deng J, Fan J. Implementation of Monte Carlo dose calculation for Cyberknife treatment planning. J Phys: Conf Ser 2008;102:1-10.

[4] Echner GG, Kilby W, Lee M, Earnst E, Sayeh S, et al. The design, physical properties and clinical utility of an iris collimator for robotic radiosurgery. Phys Med Biol 2009;54:5359-80.

[5] Heidorn SC, Kilby W, Fürweger C. Novel Monte Carlo dose calculation algorithm for robotic radiosurgery with multi leaf collimator: dosimetric evaluation. Phys Med 2018;55:25-32.

[6] Stanley J, Breitman K, Dunscombe P, Spencer DP, Lau H. Evaluation of stereotactic radiosurgery conformity indices for 170 target volumes in patients with brain metastasis. J Appl Clin Med Phys 2011;12(2):245-53.

[7] Moustakis C, Chan MKH, Kim J, Nilsson J, Bergman A, et al. Treatment planning for spinal radiosurgery. A competitive multiplatform benchmark challenge. Strahlenther Onkol 2018;194:843-54.

[8] Wang Z, Kong QT, Jing L, Wu XH, Li B, et al. Clinical outcomes of cyberknife stereotactic radiosurgery for lung metastases. J Thorac Dis 2015;7(3):407-12.

[9] Jereczek-Fossa BA, Rojas DP, Zerini D, Fodor C, Viola A, et al. Reirradiation for isolated local recurrence of prostate cancer: Mono-institutional series of 64 patients treated with slavage stereotactic body radiotherapy (SBRT). Br J Radiol 2019;91:20180494.

[10] Masi L, Zani M, Doro R, Calusi S, Cataldo Di, et al. Cyberknife MLC-based treatment planning for abdominal and pelvic SBRT: Analysis of multiple dosimetric parameters, overall scoring index and clinical scoring. Phys Med 2018;56:25-33.

[11] Chen Q, Lu W, Chen Y, Chen M, Henderson D, et al. Validation of GPU based TomoTherapy dose calculation engine. Med Phys 2012;39(4):1877-86.

[12] Shepard DM, Olivera GH, Reckwerdt PJ, Mackie TR. Iterative approaches to dose optimization in tomotherapy. Phys Med Biol 2000;45:69-90.

[13] Blanck O, Wang L, Baus W, Grimm J, Lacornerie T, et al. Inverse treatment planning for spinal robotic radiosurgery: an international multi-institutional benchmark trial. J Appl Clin Med Phys 2016;17(3):313-25.

[14] Sio TT, Jang S, Lee SW, Curran B, Pyakuryai AP, et al. Comparing Gamma Knife and CyberKnife in patients with brain metastasis. J Appl Clin Med Phys 2014;15(1):14-26.

[15] van't Riet A, Mak ACA, Moerland MA, Elders LH, van der Zee W. A conformation number to quantify the degree of conformality in brachytherapy and external beam irradiation: application to the prostate. Int J Radiat Oncol Biol Phys 1997;37(3):731-6.

[16] Paddick I, Lippitz B. A simple dose gradient measurement tool to complement the conformity index. J Neurosurg 2006;105:194-201.

[17] Bindhu J, Sanjay SS, Aruna R. Cyberknife: A double edged sword? Rep Pract Oncol Radiother 2010;15:93-7.

[18] Benedict SH, Yenice KM, Followill D, Galvin JM, Hinson W, et al. Stereotactic body radiation therapy: the report of AAPM Task Group 101. Med Phys 2010;37(8):4078-101. 
[19] McLaughlin PW, Troyer S, Berri S, Narayana V, Meirowitz A, et al. Functional anatomy of the prostate: implications for treatment planning. Int J Rad Oncol Biol Phys 2005;63(2):479-91.

[20] Pantelis E, Moutsatsos A, Zourari K, Petrokokkinos L, Sakelliou L, et al. On the output factor measuremens of the Cyberknife iris collimator small fields: experimental determination of the $\mathrm{k}$ correction factors for microchamber and diode detectors. Med Phys 2012;39(8):4875-85.

[21] Minniti G, Clarke E, Lanzetta G, Falchetto Osti M, Trasimeni G, et al. Stereotactic radiosurgery for brain metastasis: analysis of outcome and risk of brain radionecrosis. Radiat Oncol 2011;6:48. 